\title{
Review: stretching before or after exercise does not prevent muscle soreness or reduce risk of injury
}

\section{Herbert RD, Gabriel M. Effects of stretching before and after exercising on muscle soreness and risk of injury: systematic}

review. BMJ 2002;325:468-70.

\section{QUESTION: Does stretching before or after exercise reduce the risk of muscle soreness or injury and improve athletic performance?}

\section{Data sources}

Studies were identified by searching Medline (1966 to February 2000), EMBASE/Excerpta Medica (1988 to February 2000), CINAHL (1982 to January 2000), SPORTDiscus (1949-99), and PEDro (to February 2000); and reviewing bibliographies of retrieved studies.

\section{Study selection}

English language studies were selected if they were randomised or quasi-randomised controlled trials assessing the effects of any stretching technique (done immediately before or after exercising) on muscle soreness, risk of injury, or athletic performance.

\section{Data extraction}

Data were extracted on study design, participant characteristics, inclusion/exclusion criteria, interventions, and outcome measures. 2 reviewers independently assessed the methodological quality of individual studies using the PEDro scale; only studies with scores $\geq 3 / 10$ were considered for analysis. Main outcomes were muscle soreness (scale scores converted to percentages of the maximum possible score and reported on a $100 \mathrm{~mm}$ analogue scale), risk of injury, and athletic performance.

\section{Main results}

9 studies met the inclusion criteria. 2 studies were not included in the analysis because of insufficient data. Only 1 small, inconclusive study assessed the effect of stretching on athletic performance; the results were not discussed. Studies had moderate methodological quality (mean PeDRO score 4.1/10).

Meta-analysis of 5 studies of healthy young adults $(\mathrm{n}=77)$ who stretched, on average, for 300-600 seconds per session, showed no effect of stretching before or after exercise on muscle soreness at 24,48 , or 72 hours (table). Meta-analysis of 2 studies of military recruits during 12 weeks of training (2630 participants, 65

\section{Effect of stretching before or after exercise v control on muscle} soreness *

\begin{tabular}{lcl} 
Muscle soreness & $\mathbf{n}$ & $\begin{array}{l}\text { Pooled mean difference } \\
\text { between groups }(95 \% \mathrm{Cl}) \dagger\end{array}$ \\
\hline At 24 hours & 77 & $-0.9 \mathrm{~mm}(-4.4$ to 2.6$)$ \\
\hline At 48 hours & 77 & $0.3 \mathrm{~mm}(-4.0$ to 4.5$)$ \\
\hline At 72 hours & 67 & $-1.6 \mathrm{~mm}(-5.9$ to 2.6$)$ \\
\hline
\end{tabular}

${ }^{*} \mathrm{Cl}$ defined in glossary.

†Measured on a $100 \mathrm{~mm}$ visual analogue scale; negative values favour stretching. Analysis based on a fixed effects model. All mean differences are statistically non-significant. platoons) found no effect of stretching on risk of injury (pooled hazard ratio 0.95 , CI 0.78 to 1.16 ).

\section{Conclusion}

Stretching before or after exercise does not prevent muscle soreness or reduce risk of injury.

\section{COMMENTARY}

The review by Herbert and Gabriel shows that stretching before and after exercise has no protective effect on muscle soreness or risk of injury. This contradicts findings from observational studies that stretching is beneficial ${ }^{1}$ and may come as a surprise for enthusiasts who advocate stretching. A strength of this review is that all of the primary studies in the meta-analysis consistently found no effect. Data from a total of 77 participants allocated to 3 intervention arms may have limited the power to conclude that there is not even a moderate protective effect of stretching for muscle soreness. However, 2 cluster randomised studies that included a total of 2630 military recruits also found no benefit in terms of injury prevention. One might argue that injury is a more important outcome than soreness.

The difficulty of ensuring blinding of participants may have had some effect on the findings. Participants in the control group who were aware that they were not in a stretching group could have chosen to do stretching contrary to study protocol. It is possible to attempt to blind participants in such studies by not disclosing what the intervention group is receiving and by giving the control group a different activity.

Other reasons for promoting stretching include improvement in performance. ${ }^{1}$ People of different ages may place different values on performance and injury outcomes. The review by Herbert and Gabriel only dealt with younger people. However, older people can also reap the health benefits of exercise and may be more incapacitated by injury. Thus, older people may be more interested in the health benefits of exercise and less concerned with performance Studies have shown that people who continue to be physically active later in life have reductions in premature mortality. ${ }^{2}$ In this instance, time might be better spent actually doing the physical activity rather than preparing for it with stretching.

Bruce Arroll, MBChB, MHSc, $\mathrm{PhD}$ University of Auckland Auckland, New Zealand

1 American College of Sports Medicine Position Stand. The recommended quantity and quality of exercise for developing and maintaining cardiorespiratory and muscular fitness, and flexibility in healthy adults. Med Sci Sports Exerc 1998;30:975-91

2 Blair SN, Cheng Y, Holder JS. Is physical activity or physical fitness more important in defining health benefits? Med Sci Sports Exerc 2001:33:S379-99.

R.Herbert@fhs.usyd.edu. 